\title{
Holder inequalities for a subclass of univalent functions involving Dziok-Srivastava operator
}

\author{
G. Murugusundaramoorthy *, K. Vijaya, K. Deepa \\ School of Advanced Sciences, VIT University, Vellore 632014, Tamilnadu, India \\ *Corresponding author Email: gmsmoorthy@yahoo.com
}

\begin{abstract}
In this paper, we introduce a new subclasses of univalent functions defined in the open unit disc involving DziokSrivastava Operator . The results on modified Hadamard product ,Holder inequalities and closure properties under integral transforms are discussed.
\end{abstract}

Keywords: Analytic, coefficient bounds, convolution properties, convex functions, starlike functions, Holder inequality, univalent .

\section{Introduction}

Denote by $\mathcal{A}$ the class of functions of the form

$$
f(z)=z+\sum_{n=2}^{\infty} a_{n} z^{n}
$$

which are analytic in the open unit $\operatorname{disc} \mathcal{U}=\{z: z \in \mathbb{C}$ and $|z|<1\}$. Further, by $\mathcal{S}$ we shall denote the class of all functions in $\mathcal{A}$ which are normalized by $f(0)=0=f^{\prime}(0)-1$ and univalent in $\mathcal{U}$. Some of the important and wellinvestigated subclasses of the univalent function class $\mathcal{S}$ include (for example) the class $\mathcal{S}^{*}(\alpha)$ of starlike functions of order $\alpha(0 \leq \alpha<1)$ in $\mathcal{U}$ and the class $\mathcal{K}(\alpha)$ of convex functions of order $\alpha(0 \leq \alpha<1)$ in $\mathcal{U}$ satisfying the analytical criteria $\Re\left(\frac{z f^{\prime}(z)}{f(z)}\right)>\alpha$ and $\Re\left(1+\frac{z f^{\prime \prime}(z)}{f^{\prime}(z)}\right)>\alpha$ respectively.It is of interest to note that $f \in \mathcal{K}(\alpha) \Longleftrightarrow z f^{\prime} \in \mathcal{S}^{*}(\alpha)$.

For given two functions $f, g \in \mathcal{A}$ where $f(z)=z+\sum_{n=2}^{\infty} a_{n} z^{n}, \quad$ and $\quad g(z)=z+\sum_{n=2}^{\infty} b_{n} z^{n}$. The Hadamard product (or convolution) $f(z) * g(z)$ is defined $f(z) * g(z)=(f * g)(z)=z+\sum_{n=2}^{\infty} a_{n} b_{n} z^{n}, \quad z \in \mathcal{U}$.

Also denote by $\mathcal{T}$ the subclass of $\mathcal{S}$ consisting of functions of the form

$$
f(z)=z-\sum_{n=2}^{\infty} a_{n} z^{n}, \quad a_{n} \geq 0, z \in \mathcal{U}
$$

studied extensively by Silverman[11].

For positive real values of $\alpha_{1}, \ldots, \alpha_{l}$ and $\beta_{1}, \ldots, \beta_{m}\left(\beta_{j} \neq 0,-1, \ldots ; j=1,2, \ldots, m\right)$ the generalized hypergeometric function ${ }_{l} F_{m}(z)$ is defined by

$$
\begin{aligned}
{ }_{l} F_{m}(z) \equiv{ }_{l} F_{m}\left(\alpha_{1}, \ldots \alpha_{l} ; \beta_{1}, \ldots, \beta_{m} ; z\right) & :=\sum_{n=0}^{\infty} \frac{\left(\alpha_{1}\right)_{n} \ldots\left(\alpha_{l}\right)_{n}}{\left(\beta_{1}\right)_{n} \ldots\left(\beta_{m}\right)_{n}} \frac{z^{n}}{n !} \\
\left(l \leq m+1 ; l, m \in N_{0}\right. & :=N \cup\{0\} ; z \in U)
\end{aligned}
$$

where $N$ denotes the set of all positive integers and $(\lambda)_{k}$ is the Pochhammer symbol defined by

$$
(\lambda)_{n}=\left\{\begin{array}{lr}
1, & n=0 \\
\lambda(\lambda+1)(\lambda+2) \ldots(\lambda+n-1), & n \in N
\end{array}\right.
$$


The notation ${ }_{l} F_{m}$ is quite useful for representing many well-known functions such as the exponential, the Binomial, the Bessel, the Laguerre polynomial (for example see [5]).

Let $\mathcal{H}\left(\alpha_{1}, \ldots \alpha_{l} ; \beta_{1}, \ldots, \beta_{m}\right): \mathcal{A} \rightarrow \mathcal{A}$ be a linear operator defined by

$$
\begin{aligned}
\mathcal{H}\left(\alpha_{1}, \ldots \alpha_{l} ; \beta_{1}, \ldots, \beta_{m}\right) f(z) & :=z_{l} F_{m}\left(\alpha_{1}, \alpha_{2}, \ldots \alpha_{l} ; \beta_{1}, \beta_{2} \ldots, \beta_{m} ; z\right) * f(z) \\
& =z+\sum_{n=2}^{\infty} \Gamma_{n} a_{n} z^{n}
\end{aligned}
$$

where

$$
\Gamma_{n}=\frac{\left(\alpha_{1}\right)_{n-1} \ldots\left(\alpha_{l}\right)_{n-1}}{\left(\beta_{1}\right)_{n-1} \ldots\left(\beta_{m}\right)_{n-1}} \frac{1}{(n-1) !},
$$

(unless otherwise stated). For notational simplicity, we can use a shorter notation $\mathcal{H}_{m}^{l}\left[\alpha_{1}\right]$ for $\mathcal{H}\left(\alpha_{1}, \ldots \alpha_{l} ; \beta_{1}, \ldots, \beta_{m}\right)$ in the sequel. The linear operator $\mathcal{H}_{m}^{l}\left[\alpha_{1}\right]$ is called Dziok-Srivastava operator (see [5]), includes (as its special cases) various other linear operators introduced and studied by Carlson and Shaffer [3], Ruscheweyh [10] and OwaSrivastava [9]. Motivated by earlier works of Aouf et al.,[1] and Dziok and Raina[6] we define the following new subclass of $\mathcal{T}$ involving hypergeometric functions.

For $0 \leq \lambda \leq 1,0<\beta \leq 1,-1 \leq B<A \leq 1,0 \leq \gamma \leq 1$,we let $\mathcal{H F}_{\gamma}^{\lambda}(\alpha, \beta, A, B)$ denote the subclass of $\mathcal{T}$ consisting of functions $f(z)$ of the form (2) satisfying the analytic condition.

$$
\left|\frac{\frac{z F_{\lambda}^{\prime}(z)}{F_{\lambda}(z)}-1}{(B-A) \gamma\left[\frac{z F_{\lambda}^{\prime}(z)}{F_{\lambda}(z)}-\alpha\right]-B\left[\frac{z F_{\lambda}^{\prime}(z)}{F_{\lambda}(z)}-1\right]}\right|<\beta, \quad z \in U
$$

where

$$
\frac{z F_{\lambda}^{\prime}(z)}{F_{\lambda}(z)}=\frac{z \mathcal{H} f^{\prime}(z)+\lambda z^{2} \mathcal{H} f^{\prime \prime}(z)}{(1-\lambda) \mathcal{H} f(z)+\lambda z \mathcal{H} f^{\prime}(z)}, \quad 0 \leq \lambda \leq 1
$$

and

$$
\mathcal{H} f(z)=z+\sum_{n=2}^{\infty} a_{n} \Gamma_{n} z^{n}
$$

where $\Gamma_{n}$ is given by (6).

In our present investigation, we discuss some interesting properties of functions $f(z) \in \mathcal{H} \mathcal{F}_{\gamma}^{\lambda}(\alpha, \beta, A, B)$ based on convolution. Further we discuss certain closure properties under integral transformation.

In the following theorem we obtain necessary and sufficient conditions for functions $f(z) \in \mathcal{H} \mathcal{F}_{\gamma}^{\lambda}(\alpha, \beta, A, B)$.

Theorem 1.1. A function $f(z)$ of the form (2) is in the class $\mathcal{H F}_{\gamma}^{\lambda}(\alpha, \beta, A, B)$ if and only if

$$
\sum_{n=2}^{\infty} C_{n} a_{n} \leq(1-\alpha)(B-A) \beta \gamma
$$

where

$$
C_{n}=(1+n \lambda-\lambda)[(n-1)(1-\beta B)+\beta \gamma(B-A)(1-\alpha)] \Gamma_{n}
$$

and $\Gamma_{n}$ is defined by (6).

Proof. For $|z|=1$, we have

$$
\left|\frac{\frac{z F_{\lambda}^{\prime}(z)}{F_{\lambda}(z)}-1}{(B-A) \gamma\left[\frac{z F_{\lambda}^{\prime}(z)}{F_{\lambda}(z)}-\alpha\right]-B\left[\frac{z F_{\lambda}^{\prime}(z)}{F_{\lambda}(z)}-1\right]}\right|<\beta, \quad z \in \mathcal{U} .
$$

It is suffices to show that

$$
\left|z F_{\lambda}^{\prime}(z)-F_{\lambda}(z)\right|-\beta\left|(B-A) \gamma\left[z F_{\lambda}^{\prime}(z)-\alpha F_{\lambda}(z)\right]-B\left[z F_{\lambda}^{\prime}(z)-F_{\lambda}(z)\right]\right|
$$




$$
\begin{aligned}
= & \left|\sum_{n=2}^{\infty}(1+n \lambda-\lambda)(n-1) a_{n} \Gamma_{n} z^{n-1}\right| \\
& \left.\quad-\beta \mid(B-A)(1-\alpha) \gamma+\sum_{n=2}^{\infty}(1+n \lambda-\lambda)[(B-A)(n-\alpha) \gamma-B(n-1)] \Gamma_{n} a_{n} z^{n-1}\right] \mid \\
\leq & \sum_{n=2}^{\infty}(1+n \lambda-\lambda)[(n-1)(1-\beta B)+\beta \gamma(B-A)(n-\alpha)] \Gamma_{n} a_{n}-\beta \gamma(B-A)(1-\alpha) \\
\leq & 0, \text { by hypothesis. }
\end{aligned}
$$

Thus by maximum modulus theorem $f \in \mathcal{H F}_{\gamma}^{\lambda}(\alpha, \beta, A, B)$.

On the other hand suppose that

$$
\begin{aligned}
& \left|\frac{\frac{z F_{\lambda}^{\prime}(z)}{F_{\lambda}(z)}-1}{(B-A) \gamma\left[\frac{z F_{\lambda}^{\prime}(z)}{F_{\lambda}(z)}-\alpha\right]-B\left[\frac{z F_{\lambda}^{\prime}(z)}{F_{\lambda}(z)}-1\right]}\right| \\
& =\left|\frac{\frac{\sum_{n=2}^{\infty}(1+n \lambda-\lambda)(n-1) a_{n} \Gamma_{n} z^{n}}{F_{\lambda}(z)}}{\frac{(B-A) \gamma\left[z(1-\alpha)+\sum_{n=2}^{\infty}(1+n \lambda-\lambda)(n-\alpha) a_{n} \Gamma_{n} z^{n}\right]-B\left[\sum_{n=2}^{\infty}(1+n \lambda-\lambda)(n-1) a_{n} \Gamma_{n} z^{n}\right]}{F_{\lambda}(z)}}\right|<\beta .
\end{aligned}
$$

Since $\Re(z)<|z|$ for all $z$, we have

$$
\Re\left(\frac{\sum_{n=2}^{\infty}(n-1)(1+n \lambda-\lambda) \Gamma_{n} a_{n}|z|^{n-1}}{(B-A)(1-\alpha) \gamma-\sum_{n=2}^{\infty}(1+n \lambda-\lambda)[(B-A)(n-\alpha) \gamma-B(n-1)] \Gamma_{n} a_{n}|z|^{n-1}}\right)<\beta .
$$

Choosing the value of $z$ on the real axis so that $f^{\prime}(z)$ is real and letting $z \rightarrow 1^{-}$, we obtain

$$
\sum_{n=2}^{\infty}(1+n \lambda-\lambda)[(n-1)(1-\beta B)+\beta \gamma(B-A)(n-\alpha)] \Gamma_{n} a_{n} \leq \beta \gamma(B-A)(1-\alpha)
$$

and hence the proof is complete.

\section{Convolution properties for functions in the class $\mathcal{H F}_{\gamma}^{\lambda}(\alpha, \beta, A, B)$.}

In the following section, using the techniques of Schild and Silverman[12] we discuss some convolution properties for functions $f(z) \in \mathcal{H F}_{\gamma}^{\lambda}(\alpha, \beta, A, B)$.

Let the functions $f_{j}(z)(j=1,2)$ be defined by

$$
f_{j}(z)=z-\sum_{n=2}^{\infty} a_{n, j} z^{n} \quad\left(a_{n, j} \geq 0 ; j=1,2\right)
$$

then the modified Hadamard product of $f_{1}(z)$ and $f_{2}(z)$ is given by

$$
\left(f_{1} * f_{2}\right)(z)=z-\sum_{n=2}^{\infty} a_{n, 1} a_{n, 2} z^{n}
$$

Theorem 2.1. Let the function $f_{1}(z)$ defined by (14) be in the class $\mathcal{H} \mathcal{F}_{\gamma}^{\lambda}\left(\xi_{1}, \beta, A, B\right)$ and the function $f_{2}(z)$ defined by (14) be in the class $\mathcal{H F}_{\gamma}^{\lambda}\left(\xi_{2}, \beta, A, B\right)$. If the sequence $\left\{C_{n}\right\}$ is non-decreasing then $\left(f_{1} * f_{2}\right)(z) \in \mathcal{H} \mathcal{F}_{\gamma}^{\lambda}\left(\alpha^{*}, \beta, A, B\right)$ where

$$
\begin{array}{r}
\alpha^{*}=1-\frac{\left(1-\xi_{1}\right)\left(1-\xi_{2}\right)[1-\beta B+\beta \gamma(B-A)] \beta \gamma(B-A)}{(1+\lambda) \Lambda\left(\beta, \gamma, \xi_{1}, 2\right) \Lambda\left(\beta, \gamma, \xi_{2}, 2\right) \Gamma_{2}-[\beta \gamma(B-A)]^{2}\left(1-\xi_{1}\right)\left(1-\xi_{2}\right)}, \\
\Lambda\left(\beta, \gamma, \xi_{1}, 2\right)=\left[(1-\beta B)+\beta \gamma(B-A)\left(2-\xi_{1}\right)\right]
\end{array}
$$

and

$$
\Lambda\left(\beta, \gamma, \xi_{2}, 2\right)=\left[(1-\beta B)+\beta \gamma(B-A)\left(2-\xi_{2}\right)\right]
$$


Proof. In view of Theorem1.1 it is enough to show that

$$
\sum_{n=2}^{\infty} \frac{(1+n \lambda-\lambda)\left[(n-1)(1-\beta B)+\beta \gamma(B-A)\left(n-\alpha^{*}\right)\right]}{\beta \gamma(B-A)\left(1-\alpha^{*}\right)} \Gamma_{n} a_{n, 1} a_{n, 2} \leq 1 .
$$

where $\alpha^{*}$ is defined by $(16)$.

Since $f_{1} \in \mathcal{H} \mathcal{F}_{\gamma}^{\lambda}\left(\xi_{1}, \beta, A, B\right)$ we have

$$
\sum_{n=2}^{\infty} \frac{(1+n \lambda-\lambda) \Lambda\left(\beta, \gamma, \xi_{1}, n\right)}{\beta \gamma(B-A)\left(1-\xi_{1}\right)} \Gamma_{n} a_{n, 1} \leq 1
$$

and for $f_{2} \in \mathcal{H} \mathcal{F}_{\gamma}^{\lambda}\left(\xi_{2}, \beta, A, B\right)$ we have

$$
\sum_{n=2}^{\infty} \frac{(1+n \lambda-\lambda) \Lambda\left(\beta, \gamma, \xi_{2}, n\right)}{\beta \gamma(B-A)\left(1-\xi_{2}\right)} \Gamma_{n} a_{n, 2} \leq 1
$$

where

$$
\Lambda\left(\beta, \gamma, \xi_{1}, n\right)=\left[(n-1)(1-\beta B)+\beta \gamma(B-A)\left(n-\xi_{1}\right)\right]
$$

and

$$
\Lambda\left(\beta, \gamma, \xi_{2}, n\right)=\left[(n-1)(1-\beta B)+\beta \gamma(B-A)\left(n-\xi_{2}\right)\right] .
$$

On the other hand, under the hypothesis and by the Cauchy's-Schwarz inequality that

$$
\sum_{n=2}^{\infty} \frac{\left[\Lambda\left(\beta, \gamma, \xi_{1}, n\right)\right]^{1 / 2}\left[\Lambda\left(\beta, \gamma, \xi_{1}, n\right)\right]^{1 / 2}}{\sqrt{\left(1-\xi_{1}\right)\left(1-\xi_{2}\right)}}(1+n \lambda-\lambda) \Gamma_{n} \sqrt{a_{n, 1} a_{n, 2}} \leq 1
$$

From (18) and (19), it follows that

$$
\sum_{n=2}^{\infty} \frac{(1+n \lambda-\lambda)^{2} \Lambda\left(\beta, \gamma, \xi_{1}, n\right) \Gamma_{n} \Lambda\left(\beta, \gamma, \xi_{2}, n\right) \Gamma_{n}}{[\beta \gamma(B-A)]^{2}\left(1-\xi_{1}\right)\left(1-\xi_{2}\right)} a_{n, 1} a_{n, 2} \leq 1
$$

Now we have to find largest $\alpha^{*}$ such that,

$$
\begin{aligned}
& \sum_{n=2}^{\infty} \frac{(1+n \lambda-\lambda)\left[(n-1)(1-\beta B)+\beta \gamma(B-A)\left(n-\alpha^{*}\right)\right]}{\beta \gamma(B-A)\left(1-\alpha^{*}\right)} \Gamma_{n} a_{n, 1} a_{n, 2} \\
\leq & \sum_{n=2}^{\infty} \frac{(1+n \lambda-\lambda)\left[\Lambda\left(\beta, \gamma, \xi_{1}, n\right)\right]^{1 / 2}\left[\Lambda\left(\beta, \gamma, \xi_{2}, n\right)\right]^{1 / 2} \Gamma_{n}}{[\beta \gamma(B-A)] \sqrt{\left(1-\xi_{1}\right)\left(1-\xi_{2}\right)}} \sqrt{a_{n, 1} a_{n, 2}} .
\end{aligned}
$$

Or, equivalently that

$$
\sqrt{a_{n, 1} a_{n, 2}} \leq \frac{1-\alpha^{*}}{\sqrt{\left(1-\xi_{1}\right)\left(1-\xi_{2}\right)}} \frac{\left[\Lambda\left(\beta, \gamma, \xi_{1}, n\right)\right]^{1 / 2}\left[\Lambda\left(\beta, \gamma, \xi_{2}, n\right)\right]^{1 / 2}}{\left[\Lambda\left(\beta, \gamma, \alpha^{*}, n\right)\right]}, \quad(n \geq 2)
$$

where $\Lambda\left(\beta, \gamma, \alpha^{*}, n\right)=(1-B \beta)(n-1)+\beta \gamma(B-A)\left(n-\alpha^{*}\right)$.

In view of $(20)$ it is sufficient to find largest $\alpha^{*}$ such that

$$
\frac{[\beta \gamma(B-A)] \sqrt{\left(1-\xi_{1}\right)\left(1-\xi_{2}\right)}}{(1+n \lambda-\lambda)\left[\Lambda\left(\beta, \gamma, \xi_{1}, n\right)\right]^{1 / 2}\left[\Lambda\left(\beta, \gamma, \xi_{2}, n\right)\right]^{1 / 2} \Gamma_{n}} \leq \frac{1-\alpha^{*}}{\sqrt{\left(1-\xi_{1}\right)\left(1-\xi_{2}\right)}} \frac{\left[\Lambda\left(\beta, \gamma, \xi_{1}, n\right)\right]^{1 / 2}\left[\Lambda\left(\beta, \gamma, \xi_{2}, n\right)\right]^{1 / 2}}{\left[\Lambda\left(\beta, \gamma, \alpha^{*}, n\right)\right]}
$$

which yields

$$
\begin{aligned}
\alpha^{*}[(1+ & \left.n \lambda-\lambda) \Lambda\left(\beta, \gamma, \xi_{1}, n\right) \Lambda\left(\beta, \gamma, \xi_{2}, n\right) \Gamma_{n}-[\beta \gamma(B-A)]^{2}\left(1-\xi_{1}\right)\left(1-\xi_{2}\right)\right] \\
\leq & (1+n \lambda-\lambda) \Lambda\left(\beta, \gamma, \xi_{1}, n\right) \Lambda\left(\beta, \gamma, \xi_{2}, n\right) \Gamma_{n}-n[\beta \gamma(B-A)]^{2}\left(1-\xi_{1}\right)\left(1-\xi_{2}\right) \\
& -\beta \gamma(n-1)(1-\beta B)(B-A)\left(1-\xi_{1}\right)\left(1-\xi_{2}\right) .
\end{aligned}
$$

That is,

$$
\alpha^{*} \leq 1-\frac{\left[n[\beta \gamma(B-A)]^{2}-\beta \gamma(1-\beta B)(B-A)+[\beta \gamma(B-A)]^{2}\right]\left(1-\xi_{1}\right)\left(1-\xi_{2}\right)}{(1+n \lambda-\lambda) \Lambda\left(\beta, \gamma, \xi_{1}, n\right) \Lambda\left(\beta, \gamma, \xi_{2}, n\right) \Gamma_{n}-[\beta \gamma(B-A)]^{2}\left(1-\xi_{1}\right)\left(1-\xi_{2}\right)} .
$$


Let

$$
\Phi(n)=\frac{\left[n[\beta \gamma(B-A)]^{2}-(n-1) \beta \gamma(1-\beta B)(B-A)+[\beta \gamma(B-A)]^{2}\right]\left(1-\alpha_{1}\right)\left(1-\alpha_{2}\right)}{(1+n \lambda-\lambda) \Lambda\left(\beta, \gamma, \xi_{1}, n\right) \Lambda\left(\beta, \gamma, \xi_{2}, n\right) \Gamma_{n}-[\beta \gamma(B-A)]^{2}\left(1-\xi_{1}\right)\left(1-\xi_{2}\right)} .
$$

Since $\Phi(n)$ is non decreasing function of $n(n \geq 2)$, then we have $\alpha^{*} \leq 1-\Phi(2)$.

That is ,

$$
\alpha^{*} \leq 1-\frac{\left(1-\xi_{1}\right)\left(1-\xi_{2}\right)[1-\beta B+\beta \gamma(B-A)] \beta \gamma(B-A)}{(1+\lambda) \Lambda\left(\beta, \gamma, \xi_{1}, 2\right) \Lambda\left(\beta, \gamma, \alpha_{2}, 2\right) \Gamma_{2}-[\beta \gamma(B-A)]^{2}\left(1-\xi_{1}\right)\left(1-\xi_{2}\right)}
$$

and hence the proof is complete.

Remark 2.2. When $\alpha_{1}=\alpha=\alpha_{2}$, we have

$$
\alpha^{*} \leq 1-\frac{(1-\alpha)^{2}[1-\beta B+\beta \gamma(B-A)] \beta \gamma(B-A)}{(1+\lambda)[1-\beta B+\beta \gamma(B-A)(2-\alpha)]^{2} \Gamma_{2}-[\beta \gamma(B-A)]^{2}(1-\alpha)^{2}}
$$

Theorem 2.3. Let the function $f_{j}(z)(j=1,2)$ defined by (14) be in the class $\mathcal{H F}_{\gamma}^{\lambda}(\alpha, \beta, A, B)$. If the sequence $\left\{C_{n}\right\}$ is non-decreasing. Then the function

$$
h(z)=z-\sum_{n=2}^{\infty}\left(a_{n, 1}^{2}+a_{n, 2}^{2}\right) z^{n}
$$

belongs to the class $\mathcal{H F}_{\gamma}^{\lambda}(\delta, \beta, A, B)$ where

$$
\delta=1-\frac{2(1-\alpha)^{2}[1-\beta B+\beta \gamma(B-A)] \beta \gamma(B-A)}{(1+\lambda)[1-\beta B+\beta \gamma(B-A)(2-\alpha)]^{2} \Gamma_{2}-2(1-\alpha)^{2}[\beta \gamma(B-A)]^{2}} .
$$

Proof. By virtue of Theorem 1.1, it is sufficient prove that

$$
\sum_{n=2}^{\infty} \frac{(1+n \lambda-\lambda)[(n-1)(1-\beta B)+\beta \gamma(B-A)(n-\delta)] \Gamma_{n}}{\beta \gamma(B-A)(1-\delta)}\left(a_{n, 1}^{2}+a_{n .2}^{2}\right) \leq 1 .
$$

Since $f_{j}(z)(j=1,2) \in \mathcal{H F}_{\gamma}^{\lambda}(\alpha, \beta, A, B)$ we have

$$
\begin{aligned}
\sum_{n=2}^{\infty} & \left\{\frac{(1+n \lambda-\lambda)[(n-1)(1-\beta B)+\beta \gamma(B-A)(n-\alpha)] \Gamma_{n}}{\beta \gamma(B-A)(1-\alpha)}\right\}^{2} a_{n, 1}^{2} \\
& \leq \sum_{n=2}^{\infty}\left\{\frac{(1+n \lambda-\lambda)[(n-1)(1-\beta B)+\beta \gamma(B-A)(n-\alpha)] \Gamma_{n} a_{n, 1}}{\beta \gamma(B-A)(1-\alpha)}\right\}^{2} \leq 1
\end{aligned}
$$

and

$$
\begin{aligned}
\sum_{n=2}^{\infty} & \left\{\frac{(1+n \lambda-\lambda)[(n-1)(1-\beta B)+\beta \gamma(B-A)(n-\alpha)] \Gamma_{n}}{\beta \gamma(B-A)(1-\alpha)}\right\}^{2} a_{n, 2}^{2} \\
& \leq \sum_{n=2}^{\infty}\left\{\frac{(1+n \lambda-\lambda)[(n-1)(1-\beta B)+\beta \gamma(B-A)(n-\alpha)] \Gamma_{n} a_{n, 2}}{\beta \gamma(B-A)(1-\alpha)}\right\}^{2} \leq 1 .
\end{aligned}
$$

It follows from (25) and (26) that

$$
\sum_{n=2}^{\infty} \frac{1}{2}\left\{\frac{(1+n \lambda-\lambda)[(n-1)(1-\beta B)+\beta \gamma(B-A)(n-\alpha)] \Gamma_{n}}{\beta \gamma(B-A)(1-\alpha)}\right\}^{2}\left(a_{n, 1}^{2}+a_{n, 2}\right) \leq 1 .
$$

Therefore we need to find the largest $\delta$, such that

$$
\begin{aligned}
& \frac{(1+n \lambda-\lambda)[(n-1)(1-\beta B)+\beta \gamma(B-A)(n-\delta)] \Gamma_{n}}{\beta \gamma(B-A)(1-\delta)} \\
& \quad \leq \frac{1}{2}\left[\frac{(1+n \lambda-\lambda)[(n-1)(1-\beta B)+\beta \gamma(B-A)(n-\alpha)] \Gamma_{n}}{\beta \gamma(B-A)(1-\alpha)}\right]^{2} \quad(n \geq 2)
\end{aligned}
$$

that is

$$
\delta \leq 1-\frac{2(n-1)(1-\beta B) \beta \gamma(B-A)(1-\alpha)^{2}-2 n[\beta \gamma(B-A)]^{2}(1-\alpha)^{2}+2[\beta \gamma(B-A)]^{2}(1-\alpha)^{2}}{(1+n \lambda-\lambda)[(n-1)(1-\beta B)+\beta \gamma(B-A)(n-\delta)]^{2} \Gamma_{n}-2[\beta \gamma(B-A)]^{2}(1-\alpha)^{2}} .
$$


Since

$$
\Psi(n)=1-\frac{2(n-1)(1-\beta B) \beta \gamma(B-A)(1-\alpha)^{2}-2 n[\beta \gamma(B-A)]^{2}(1-\alpha)^{2}+2[\beta \gamma(B-A)]^{2}(1-\alpha)^{2}}{(1+n \lambda-\lambda)[(n-1)(1-\beta B)+\beta \gamma(B-A)(n-\delta)]^{2} \Gamma_{n}-2[\beta \gamma(B-A)]^{2}(1-\alpha)^{2}}
$$

is an increasing function of $n,(n \geq 2)$, we readily have

$$
\delta \leq \Psi(2)=1-\frac{2(1-\alpha)^{2}[1-\beta B+\beta \gamma(B-A)] \beta \gamma(B-A)}{(1+\lambda)[1-\beta B+\beta \gamma(B-A)(2-\alpha)]^{2} \Gamma_{2}-2(1-\alpha)^{2}[\beta \gamma(B-A)]^{2}},
$$

which completes the proof.

\section{Hölder's inequality}

Recently, Nishiwaki, Owa and Srivastava [8] have studied some results of Holder-type inequalities for a subclass of uniformly starlike functions. Now, we recall the generalization of the convolution due to Choi, Kim and Owa [4] as given below,

$$
H_{m}(z)=z-\sum_{n=2}^{\infty}\left(\prod_{j=1}^{m} a_{n, j}^{p_{j}}\right) z^{n} \quad\left(p_{j}>0, j=1,2, \ldots, m\right) .
$$

Further for functions $f_{j}(z) \in \mathcal{H F}_{\gamma}^{\lambda}(\alpha, \beta, A, B)(j=1,2, \ldots, m)$ given by (15), the familiar Holder inequality assumes the following form

$$
\sum_{n=2}^{\infty}\left(\prod_{j=1}^{m} a_{n, j}\right) \leq \prod_{j=1}^{m}\left(\sum_{n=2}^{\infty} a_{n, j}^{p_{j}}\right)^{\frac{1}{p_{j}}} \quad\left(p_{j}>1, j=1,2, \ldots, m ; \sum_{j=i}^{m} \frac{1}{p_{j}} \geq 1\right) .
$$

Theorem 3.1. (Holder's Inequality:) If $f_{j}(z) \in \mathcal{H F}_{\gamma}^{\lambda}\left(\xi_{j}, \beta, A, B\right),-1 \leq B<A \leq 1,0<\beta \leq 1,0 \leq \lambda \leq 1, j=$ $1,2, \ldots$, m then $\mathcal{H}_{m}(z) \in \mathcal{H F}_{\gamma}^{\lambda}(\xi, \beta, A, B)$ with

$$
\xi \leq 1-\frac{\prod_{j=i}^{m}\left(1-\xi_{j}\right)^{p_{j}}-[(1-\beta B)+\beta \gamma(B-A)][\beta \gamma(B-A)]^{s}}{(1+\lambda)^{s-1} \Gamma_{n}^{s-1} \prod_{j=i}^{m}\left[1-\beta B+\beta \gamma(B-A)\left(2-\xi_{j}\right)\right]^{p_{j}}-[\beta \gamma(B-A)]^{s} \prod_{j=i}^{m}\left(1-\xi_{j}\right)^{p_{j}}} .
$$

where $\left(S=\sum_{j=i}^{m} p_{j} \geq 1 ; p_{j} \geq \frac{1}{q_{j}}(j=1,2, \ldots, m), q_{j}>1(j=1,2, \ldots, m) ; \sum_{j=i}^{m} \frac{1}{q_{j}} \geq 1\right)$.

Proof. Let $f_{j}(z) \in \mathcal{H} \mathcal{F}_{\gamma}^{\lambda}\left(\xi_{j}, \beta, A, B\right)(j=1,2, \ldots, m)$. Then we have

$$
\sum_{n=2}^{\infty} \frac{(1+n \lambda-\lambda)\left[(n-1)(1-\beta B)+\beta \gamma(B-A)\left(n-\xi_{j}\right)\right]}{\beta \gamma(B-A)\left(1-\xi_{j}\right)} \Gamma_{n} a_{n, j} \leq 1
$$

which in turn, implies that

$$
\begin{gathered}
\left(\sum_{n=2}^{\infty} \frac{(1+n \lambda-\lambda)\left[(n-1)(1-\beta B)+\beta \gamma(B-A)\left(n-\xi_{j}\right)\right]}{\beta \gamma(B-A)\left(1-\xi_{j}\right)} \Gamma_{n} a_{n, j}\right)^{\frac{1}{q_{j}}} \leq 1 \\
\left(q_{j}>1 \quad(j=1,2, \ldots, m) ; \sum_{j=i}^{m} \frac{1}{q_{j}}=1\right) .
\end{gathered}
$$

Applying the Holder inequality (30), we arrive at the following inequality

$$
\sum_{n=2}^{\infty}\left[\sum_{j=i}^{m}\left(\frac{(1+n \lambda-\lambda)\left[(n-1)(1-\beta B)+\beta \gamma(B-A)\left(n-\xi_{j}\right)\right]}{\beta \gamma(B-A)\left(1-\xi_{j}\right)} \Gamma_{n}\right)^{\frac{1}{q_{j}}} a_{n, j}^{\frac{1}{q_{j}}}\right] \leq 1 .
$$

Thus, we have to determine the largest $\xi$ such that

$$
\sum_{n=2}^{\infty} \frac{(1+n \lambda-\lambda)[(n-1)(1-\beta B)+\beta \gamma(B-A)(n-\xi)]}{\beta \gamma(B-A)(1-\xi)} \Gamma_{n}\left(\prod_{j=1}^{m} a_{n, j}^{p_{j}}\right) \leq 1
$$


that is,

$$
\begin{aligned}
& \sum_{n=2}^{\infty} \frac{(1+n \lambda-\lambda)[(n-1)(1-\beta B)+\beta \gamma(B-A)(n-\xi)]}{\beta \gamma(B-A)(1-\xi)} \Gamma_{n}\left(\prod_{j=1}^{m} a_{n, j}^{p_{j}}\right) \\
\leq & \sum_{n=2}^{\infty}\left[\sum_{j=i}^{m}\left(\frac{(1+n \lambda-\lambda)\left[(n-1)(1-\beta B)+\beta \gamma(B-A)\left(n-\xi_{j}\right)\right]}{\beta \gamma(B-A)\left(1-\xi_{j}\right)} \Gamma_{n}\right)^{\frac{1}{q_{j}}} a_{n, j}^{\frac{1}{q_{j}}}\right] .
\end{aligned}
$$

Therefore, we need to find the largest $\xi$ such that

$$
\begin{aligned}
& \frac{(1+n \lambda-\lambda)[(n-1)(1-\beta B)+\beta \gamma(B-A)(n-\xi)]}{\beta \gamma(B-A)(1-\xi)} \Gamma_{n}\left(\prod_{j=1}^{m} a_{n, j}^{p_{j}}\right) \\
\leq & \prod_{j=i}^{m}\left(\frac{(1+n \lambda-\lambda)\left[(n-1)(1-\beta B)+\beta \gamma(B-A)\left(n-\xi_{j}\right)\right]}{\beta \gamma(B-A)\left(1-\xi_{j}\right)} \Gamma_{n}\right)^{\frac{1}{q_{j}}} a_{n, j}^{\frac{1}{q_{j}}} .
\end{aligned}
$$

Since,

$$
\prod_{j=i}^{m}\left(\frac{(1+n \lambda-\lambda)\left[(n-1)(1-\beta B)+\beta \gamma(B-A)\left(n-\xi_{j}\right)\right]}{\beta \gamma(B-A)\left(1-\xi_{j}\right)} \Gamma_{n}\right)^{p_{j}-\frac{1}{q_{j}}} a_{n, j}^{p_{j}-\frac{1}{q_{j}}} \leq 1,\left(p_{j}-\frac{1}{q_{j}} \geq 0, j=1,2, \ldots, m\right)
$$

we see that,

$$
\prod_{j=i}^{m} a_{n, j}^{p_{j}-\frac{1}{q_{j}}} \leq \frac{1}{\prod_{j=i}^{m}\left(\frac{(1+n \lambda-\lambda)\left[(n-1)(1-\beta B)+\beta \gamma(B-A)\left(n-\xi_{j}\right)\right]}{\beta \gamma(B-A)\left(1-\xi_{j}\right)} \Gamma_{n}\right)^{p_{j}-\frac{1}{q_{j}}}}
$$

This last inequality (31) implies that

$$
\begin{array}{r}
\frac{(1+n \lambda-\lambda)[(n-1)(1-\beta B)+\beta \gamma(B-A)(n-\xi)]}{\beta \gamma(B-A)(1-\xi)} \Gamma_{n} \\
\leq \frac{\prod_{j=i}^{m}(1+n \lambda-\lambda)^{p_{j}}\left[(n-1)(1-\beta B)+\beta \gamma(B-A)\left(n-\xi_{j}\right) \Gamma_{n}\right]^{p_{j}}}{\prod_{j=i}^{m}\left[\beta \gamma(B-A)\left(1-\xi_{j}\right)\right]^{p_{j}}}
\end{array}
$$

which implies

$$
\begin{aligned}
& {[(n-1)(1-\beta B)+\beta \gamma(B-A)(n-\xi)] \prod_{j=i}^{m}[\beta \gamma(B-A)]^{p_{j}-1}\left(1-\xi_{j}\right)^{p_{j}}} \\
& \quad \leq \prod_{j=i}^{m}(1+n \lambda-\lambda)^{p_{j}-1} \Gamma_{n}^{p_{j}-1}\left[(n-1)(1-\beta B)+\beta \gamma(B-A)\left(n-\xi_{j}\right)\right]^{p_{j}}\left(1-\xi_{j}\right)^{p_{j}}
\end{aligned}
$$

where,

$$
\xi \leq 1-\frac{n \Upsilon_{j}+\Upsilon_{j}-(n-1) 1-\beta B \prod_{j=i}^{m}[\beta \gamma(B-A)]^{p_{j}-1}\left(1-\xi_{j}\right)^{p_{j}}}{\prod_{j=i}^{m}(1+n \lambda-\lambda)^{p_{j}-1} \Gamma_{n}^{p_{j}-1}\left[(n-1)(1-\beta B)+\beta \gamma(B-A)\left(n-\xi_{j}\right)\right]^{p_{j}}-\Upsilon_{j}}
$$

and $\Upsilon_{j}=\prod_{j=i}^{m}[\beta \gamma(B-A)]^{p_{j}}\left(1-\xi_{j}\right)^{p_{j}}$.

Let

$$
\Phi(n) \leq 1-\frac{n \Upsilon_{j}+\Upsilon_{j}-(n-1) 1-\beta B \prod_{j=i}^{m}[\beta \gamma(B-A)]^{p_{j}-1}\left(1-\xi_{j}\right)^{p_{j}}}{\prod_{j=i}^{m}(1+n \lambda-\lambda)^{p_{j}-1} \Gamma_{n}^{p_{j}-1}\left[(n-1)(1-\beta B)+\beta \gamma(B-A)\left(n-\xi_{j}\right)\right]^{p_{j}}-\Upsilon_{j}}
$$

which is an increasing function in $n$, hence we have

$$
\xi \leq \Phi(2)=1-\frac{\prod_{j=i}^{m}\left(1-\xi_{j}\right)^{p_{j}}-[(1-\beta B)+\beta \gamma(B-A)][\beta \gamma(B-A)]^{s}}{(1+\lambda)^{s-1} \Gamma_{n}^{s-1} \prod_{j=i}^{m}\left[1-\beta B+\beta \gamma(B-A)\left(2-\xi_{j}\right)\right]^{p_{j}}-[\beta \gamma(B-A)]^{s} \prod_{j=i}^{m}\left(1-\xi_{j}\right)^{p_{j}}} .
$$

This completes the proof of the theorem. 


\section{Closure properties under integral transforms}

Integral transform of the class $\mathcal{H F}_{\gamma}^{\lambda}(\alpha, \beta, A, B)$. For $f \in \mathcal{S}$, we define the integral transform

$$
V_{\lambda}(f)(z)=\int_{0}^{1} \lambda(t) \frac{f(t z)}{t} d t
$$

where $\lambda$ is a real valued, non-negative weight function normalized so that $\int_{0}^{1} \lambda(t) d t=1$. Since special cases of $\lambda(t)$ are particularly interesting such as $\lambda(t)=(1+c) t^{c}, c>-1$, for which $V_{\lambda}$ is known as Bernardi Operator [2], and

$$
\lambda(t)=\frac{(c+1)^{\delta}}{\Gamma(\delta)} t^{c}\left(\log \frac{1}{t}\right)^{\delta-1}, c>-1, \delta \geq 0
$$

which gives the Komatu operator[7].

Theorem 4.1. Let $f(z) \in \mathcal{H F}_{\gamma}^{\lambda}(\alpha, \beta, A, B)$. Then $V_{\lambda}(f(z)) \in \mathcal{H F}_{\gamma}^{\lambda}(\alpha, \beta, A, B)$.

Proof. By definition, we have

$$
\begin{aligned}
& V_{\lambda}(f)(z)=\frac{(c+1)^{\delta}}{\Gamma(\delta)} \int_{0}^{1}(-1)^{\delta-1} t^{c}(\log t)^{\delta-1}\left[z-\sum_{n=2}^{\infty} a_{n} z^{n} t^{n-1}\right] d t \\
& V_{\lambda}(f)(z)=\frac{(-1)^{\delta-1}(c+1)^{\delta}}{\Gamma(\delta)} \lim _{r \rightarrow o^{+}}\left[\int_{r}^{1} t^{c}(\log t)^{\delta-1}\left[z-\sum_{n=2}^{\infty} a_{n} z^{n} t^{n-1}\right] d t\right]
\end{aligned}
$$

By simple computation, we get

$$
V_{\lambda}(f)(z)=z-\sum_{n=2}^{\infty}\left(\frac{c+1}{c+n}\right)^{\delta} a_{n} z^{n}
$$

We need to prove that $V_{\lambda}(f(z)) \in \mathcal{H} \mathcal{F}_{\gamma}^{\lambda}(\alpha, \beta, A, B)$, it is enough to prove

$$
\sum_{n=2}^{\infty} \frac{(1+n \lambda-\lambda)[(n-1)(1-\beta B)+\beta \gamma(B-A)(n-\alpha)]}{\beta \gamma(B-A)(1-\alpha)}\left(\frac{c+1}{c+n}\right)^{\delta} \Gamma_{n} a_{n} \leq 1
$$

on the other hand by Theorem 1.1, $f(z) \in \mathcal{H F}_{\gamma}^{\lambda}(\alpha, \beta, A, B)$ if and only if

$$
\sum_{n=2}^{\infty} \frac{(1+n \lambda-\lambda)[(n-1)(1-\beta B)+\beta \gamma(B-A)(n-\alpha)]}{\beta \gamma(B-A)(1-\alpha)} \Gamma_{n} a_{n} \leq 1
$$

Hence $\frac{c+1}{c+n}<1$. Therefore (32) holds and the proof is complete.

The above theorem yields the following two special cases.

Theorem 4.2. 1) If $f(z)$ is starlike of order $\gamma$ then $V_{\lambda} f(z)$ is also starlike of order $\alpha$.

2) If $f(z)$ is convex of order $\gamma$ then $V_{\lambda} f(z)$ is also convex of order $\alpha$.

Theorem 4.3. Let $f \in \mathcal{H F}_{\gamma}^{\lambda}(\alpha, \beta, A, B)$. Then $V_{\lambda} f(z)$ is starlike of order $0 \leq \xi<1$ in $|z|<R_{1}$, where

$$
R_{1}=\inf _{n}\left\{\frac{(1-\xi) C_{n}}{(n-\xi)(B-A)(1-\alpha) \beta \gamma}\right\}^{\frac{1}{n-1}}
$$

where $C_{n}$ is defined by (11).

Proof. It is sufficient to prove

$$
\left|\frac{z\left(V_{\lambda} f(z)\right)^{\prime}}{V_{\lambda} f(z)}-1\right|<1-\xi \quad \text { for } \quad|z|<R_{1},
$$


where $R_{1}$ is given by (33)

$$
\left|\frac{z\left(V_{\lambda} f(z)\right)^{\prime}}{V_{\lambda} f(z)}-1\right| \leq \frac{\sum_{n=2}^{\infty}(n-1)\left(\frac{c+1}{c+n}\right)^{\delta} a_{n}|z|^{n-1}}{1-\sum_{n=2}^{\infty}\left(\frac{c+1}{c+n}\right)^{\delta} a_{n}|z|^{n-1}}
$$

Thus

$$
\left|\frac{z\left(V_{\lambda} f(z)\right)^{\prime}}{V_{\lambda} f(z)}-1\right|<1-\xi \quad \text { if, } \sum_{n=2}^{\infty}\left(\frac{c+1}{c+n}\right)^{\delta}\left(\frac{n-\xi}{1-\xi}\right) a_{n}|z|^{n-1} \leq 1 .
$$

But by Theorem 1.1, we have

$$
\sum_{n=2}^{\infty} \frac{C_{n}}{(B-A)(1-\alpha) \beta \gamma} a_{n} \leq 1
$$

Comparing (34) and (35), we have $\left(\frac{c+1}{c+n}\right)^{\delta}\left(\frac{n-\xi}{1-\xi}\right)|z|^{n-1} \leq \frac{C_{n}}{(B-A)(1-\alpha) \beta \gamma}$. Thus

$$
|z| \leq\left\{\left(\frac{c+n}{c+1}\right)^{\delta} \frac{(1-\xi) C_{n}}{(n-\xi)(B-A)(1-\alpha) \beta \gamma}\right\}^{\frac{1}{n-1}} .
$$

Therefore, the proof is complete.We state the following theorem without proof.

Theorem 4.4. Let $f \in \mathcal{H F}_{\gamma}^{\lambda}(\alpha, \beta, A, B)$. Then $V_{\lambda} f(z)$ is convex of order $0 \leq \xi<1$ in $|z|<R_{2}$, where

$$
R_{2}=\inf _{n}\left\{\left(\frac{c+n}{c+1}\right)^{\delta} \frac{(1-\xi) C_{n}}{n(n-\xi)(B-A)(1-\alpha) \beta \gamma}\right\}^{\frac{1}{n-1}}
$$

\section{References}

[1] M. K. Aouf and G.Murugusundaramoorthy, On a subclass of uniformly convex functions defined by the Dziok-Srivastava Operator, Austral. J.Math.Anal.and appl.(AJMAA) Volume 5, Issue 1, Article 3, pp. 1-17,( 2008).

[2] S. D. Bernardi, Convex and starlike univalent functions, Trans. Amer. Math. Soc., 135 (1969), pp.429-446.

[3] B. C. Carlson and D. B. Shaffer, Starlike and prestarlike hypergeometric functions, SIAM J. Math. Anal., 15(1984), pp. $737-745$.

[4] J.H. Choi, Y.C. Kim and S. Owa, Generalizations of Hadamard products of functions with negative coefficients, J. Math. Anal. Appl., 199 (1996), pp. 495-501.

[5] J.Dziok and H.M.Srivastava, Certain subclasses of analytic functions associated with the generalized hypergeometric function, Intergral Transform and Spec. Funct., 14 (2003), pp.7 - 18.

[6] J. Dziok, and R. K. Raina (2004). Families of analytic functions associated with the Wright generalized hypergeometric function, Demonstratio Math., 37 (3), pp. 533-542.

[7] Y.C. Kim and F. Ronning, Integral transform of certain subclasses of analytic functions, J. Math. Anal. Appl., 258, (2001), pp. $466-489$.

[8] J. Nishiwaki, S. Owa and H.M. Srivastava, Convolution and Holder-type inequalities for a certain class of analytic functions, Math. Inequal. Appl., 11 (2008), pp.717-727.

[9] S.Owa and H.M.Srivastava:Univalent and Starlike generalized hypergeometric functions, Canad.J.Math.39(1987), pp.1057-1077.

[10] S.Ruscheweyh, New criteria for univalent functions, Proc. Amer. Math. Soc. 49, (1975), pp.109-115.

[11] H. Silverman, Univalent functions with negative coefficients, Proc. Amer. Math. Soc. , 51 (1975), pp.109 - 116.

[12] A. Schild and H. Silverman, Convolution of univalent functions with negative coefficients, Ann. Univ. Mariae-Curie Sk lodowska Sect.A 29 (1975), pp.99 - 107. 